\title{
Theoretical Data on Support of a Unified Indoor Geolocation Channel Model
}

\author{
Ilir F. Progri' ${ }^{1}$, William R. Michalson ${ }^{2}$, Jinling Wang ${ }^{3}$, Matthew C. Bromberg ${ }^{4}$ \\ ${ }^{1}$ Giftet Inc., 5 Euclid Ave. \#3, Worcester, MA 01610, USA \\ ORCID: 0000-0001-5197-1278 \\ ${ }^{2}$ Worcester Polytechnic Institute, Worcester, MA \\ ${ }^{3}$ University of New South Wales, Sydney, Australia \\ ${ }^{4}$ Elected Engineering, $M A$
}

Correspondence should be addressed to Ilir Progri; iprogri@giftet.com

Received March 23, 2017; Revised March 24, 31, 2017-June 7, 2017, Accepted July 16, 2017; Published November 1, 2017.

Scientific Editor-in-Chief/Editor: Ilir F. Progri

Copyright (C 2017 Ilir F. Progri et al. This is an open access article distributed under the Creative Commons Attribution License, which permits unrestricted use, distribution, and reproduction in any medium, provided the original work is properly cited.

In this paper we present theoretical data in support of the unified indoor geolocation channel model namely: path loss and multipath distribution models. First, the path loss model is currently accepted to be a function of the transmitter and receiver geometry and frequency of operation. Second, the most widely used and accepted indoor channel multipath distribution models are Nakagami with $m$ degrees of freedom, Rayleigh, Rician, and lognormal. The purpose of this paper is twofold: to provide a better interpretation of the sets of theoretical data for the indoor channel model; and, to explain the lack of fitting of the well-known multipath distribution models from the previous measurement data sets reported in the literature; thus, providing support for the unified indoor channel model theory.

The unified path loss model consists of an approach for linking together the path loss models of the three geolocation systems (macro-cellular, microcellular, and indoor) with the distance between the transmitter and receiver, $R$, and the frequency of operation, $f$. The path loss caused by the increase of the transmitter receiver distance is much more severe than the path loss caused by the increase of the frequency of operation. The bottom line here is that we need to design future receivers or propose a signal structure ${ }^{i}$ that will account for 40 to $80 \mathrm{~dB}$ of signal degradation indoors.

The unified multipath distribution consists of a linear transformation of the well-known multipath distribution models such as Nakagami with $m$ degrees of freedom, Rayleigh, Rician, and lognormal. While it is rather straight forward to prove the unified geolocation multipath distribution model when only the contributing individual distributions are Rayleigh, Rician, and lognormal, if we assume that we have a fourth distribution such as Nakagami with $m$ degrees then the process is not straight forward any more. We will investigate this and report the results in the future.

The main purpose of the unified multipath distribution model is to enable the calculations of reflections' gain. Assuming that the channel is composed of individual distributions such as Rayleigh, Rician, and Lognormal we have perform reflection gain calculations. 
From the theoretical data it appears that reflections with gain either 3, or 4, or $5 \mathrm{~dB}$ or higher are on the order of either 2.6, or 1.3, or 1.4 occurrences out of either one minute, or three minutes, or one hour. On the other hand, reflections with gain either 6 or $7 \mathrm{~dB}$ or higher are of the order of 1 out of either three days or $\sim 12$ years. And this is the most important conclusion of this work that for simulation or implementation purposes we should never consider reflections with gains up to $7 \mathrm{~dB}$ greater than the LOS gain.

Index Terms - Unified, Rayleigh, Rician, Lognormal, reflections, multipath, macro-cellular, microcellular, indoor, path loss analysis, multipath analysis.

\section{Introduction}

In the past we have proposed a theory for the unified geolocation channel models [1]-[4]. Our unified geolocation channel model theory was based on the extensive research in this area, the majority of which is presented in [1], [2].

The unified geolocation channel model consists of two models: the path loss model and the multipath distribution model.

The path loss model consists of a relationship of the path gain with the transmitter receiver distance and the frequency of operations. It is suggested [1]-[3] that the path loss gain factor is inversely proportional with the $n$th power order of the distance between the transmitter and receiver. Typical values of $n$ range from 2-6. We will call $n$ the transmitter receiver index. Obviously for open air or outdoor geolocation systems the transmitter receiver index $n$ is equal to 2 and for severe indoor geolocation systems the index $n$ has higher values close to 6 . Although higher values of $n$ may also be observed we will assume that for the purposes of this research typical values of the transmitter receiver index $n$ is close to 6 . As reported in our investigation the path loss caused by the increase of the transmitter receiver distance is much more severe than the path loss caused by the increase of the frequency of operation. The bottom line here is that we need to design future receivers or propose a signal structure ${ }^{\mathrm{ii}}$ that will account for 40 to $80 \mathrm{~dB}$ of signal degradation indoors.

While the first model denotes the line-of-sight (LOS) value of the path-loss gain, typically other paths are also observed [1]-[4]. Therefore, there is a need for a multipath distribution model. Although there are many elements in the multipath distribution model such as the path gain distribution, the interarrival times, and the path phase' distribution, or the number of observable paths distribution the most important of all these are two: the path gain distribution and the number of observable path distribution.
The main idea in this paper is to come up with a reasonable number of the path gains of the observable paths based on known distributions. In the process of doing all these we also provide a thorough discussion of the unified geolocation multipath distribution model. The purpose of this model is twofold: to provide the framework for reconciling the entire known or unknown multipath distribution models; and, to enable a procedure for providing reasonable bounds on the values of the path gains of the observable paths.

In summary this procedure will provide sufficient information on the completeness of the multipath model for all geolocation systems.

While it is rather straight forward to prove the unified geolocation multipath distribution model when only the contributing individual distributions are Rayleigh, Rician, and lognormal, if we assume that we have a fourth distribution such as Nakagami with $m$ degrees then the process is not straight forward any more. As indicated in the theoretical derivation it appears that obtaining the Nakagami with $m$ degrees of freedom parameters is not straightforward from the mean and variance values of the composite distribution. We will investigate this and report the results in the future.

The paper is organized as follows. First we describe the unified LOS path-loss (gain) model. Second we present the unified multipath distribution model. Third we perform observable path-loss gain computations. Fourth we provide the numerical, theoretical results. Fifth, we provide the conclusion.

From the theoretical data it appears that reflections with gain either 3 , or 4 , or $5 \mathrm{~dB}$ or higher are on the order of either 2.6 , or 1.3 , or 1.4 occurrences out of either one minute, or three minutes, or one hour. On the other hand, reflections with gain either 6 or $7 \mathrm{~dB}$ or higher are of the order of 1 out of either three days or $\sim 12$ years. And this is the most important conclusion of this work that for simulation or implementation purposes we should never consider reflections with gains up to $7 \mathrm{~dB}$ greater than the LOS gain 


\section{Unified LOS Path-loss (Gain) Model}

The unified path loss model consists of an approach for linking together the path loss models of the three geolocation systems (macro-outdoor, micro-outdoor, and indoor) with the distance between the transmitter and receiver, $R$, and the frequency of operation, $f$. Although there are several parameters that affect the power loss factor, we consider $R$ and $f$ as the most important parameters for two reasons. Most of the geolocation systems discussed in [1], [2] are based on a direct measure of the time of travel; i.e., distance between the transmitter and receiver. The frequency of these systems varies; hence, the path loss factor varies as a function of the frequency as well. The unified path loss equation is given by:

$$
Q[R(t), f]=Q_{0}+10 n \log _{10} \frac{R_{0}}{R(t)}+10 m \log _{10} \frac{f_{0}}{f}+N(0, \sigma)
$$

where $Q_{0}=Q\left(R_{0}, f_{0}\right)$ is the path loss corresponding to the frequency $f_{0}$ and distance $R_{0}$ and $N(0, \sigma)$ is a normal random variable.

\section{Unified Multipath Distribution Model}

Let's assume that we have three multipath distribution models: Lognormal, Rician, and Rayleigh.

Let $f_{i}\left(a_{k}\right)$ denote the multipath distribution model, which for the macro-geolocation systems $(i=1)$ (lognormal distribution), for the micro-geolocation systems $(i=2)$ (Rician distribution), and and for the indoor geolocation systems $(i=3)$ (Rayleigh distribution).

Let $f\left(a_{k}\right)$ denote the unified multipath distribution model. Let $r$ and $n_{i}$ be defined as follows:

$$
r \equiv a_{k} \text { and } 0 \leq n_{i} \leq 1
$$

subject to

$$
\sum_{i=1}^{3} n_{i}=1
$$

According to the unified distribution channel model $f(r)$ can be written as [1], [2], [4]

$$
f(r)=\sum_{i=1}^{3} n_{i} f_{i}(r)
$$

In [1], [2], [4] we provide four theorems that enables the validity of this distribution expansion.

However, here we explain the usefulness of the unified geolocation multipath distribution channel model which is twofold:

1. To provide the framework for all the available multipath distribution channel models;
2. To enable the computation of observable path gains.

To illustrate the first goal we consider three known multipath distributions: Lognormal, Rician, and Rayleigh and the resulting composite distribution with $\mu_{a}=0.6$ and $\sigma_{a}=$ 0.0626 and $n=\{0.25,0.35,0.4\}$.

Other distributions are proposed such as Nakagami with $m$ degrees of freedom [5], [7].

Let assume that $R$ is a random variable, whose probability density function is Nakagami, is given by [8]-[10]:

$$
f_{R}(r)=\mid \begin{array}{cc}
2\left(\frac{\mu}{\omega}\right)^{\mu} \frac{1}{\Gamma(\mu)} r^{(2 \mu-1)} e^{-\frac{\mu}{\omega} r^{2}}, & r \geq 0 \\
0, & r<0
\end{array}
$$

with shape parameter $\mu>0.5$ and scale parameter $\omega>0$, where

$$
\Gamma(\mu)=\int_{0}^{\infty} x^{\mu-1} e^{-x} d x, \mu>0
$$

The Nakagami $\mu>0.5$ and $\omega>0$ parameters are determined from [8]

$$
\omega=E\left[R^{2}\right] \text { and } \mu=\frac{\left(E\left[R^{2}\right]\right)^{2}}{\operatorname{Var}\left[R^{2}\right]}
$$

The expected value, $E[R]$, of the random Nakagami variable $R$ is given by (see Appendix A and B for complete derivations) $[1],[12]$

$$
E[R]=\sqrt{\frac{\omega}{\mu}} \frac{\Gamma\left(\mu+\frac{1}{2}\right)}{\Gamma(\mu)}
$$

The variance, $\operatorname{Var}[R]$, of the random Nakagami variable $R$ is given by (see Appendix A and $\mathrm{B}$ for complete derivations) [1], [12]

$$
\operatorname{Var}[R]=\omega\left[1-\frac{\Gamma^{2}\left(\mu+\frac{1}{2}\right)}{\mu \Gamma^{2}(\mu)}\right]
$$

It is rather simple to obtain numerical values of the mean and the variance knowing $\mu>0.5$ and $\omega>0$ parameters. However, in order to apply the unified multipath distribution model theory we need to determine the parameters $\mu>0.5$ and $\omega>0$ from the mean and the variance values which is rather difficult in a closed form expression.

If we assume that $m=\mu>0.5$ is an integer than we obtain the probability density function of the Nakagami with $m$ degrees of freedom is given by:

$$
f_{R}(r)=\mid \begin{array}{cc}
2\left(\frac{m}{\omega}\right)^{m} \frac{1}{\Gamma(m)} r^{(2 m-1)} e^{-\frac{m}{\omega} r^{2}}, & r \geq 0 \\
0, & r<0
\end{array}
$$

From where we obtain the mean [11] 
$E[R]=\sqrt{\frac{\omega}{m}} \frac{\Gamma\left(m+\frac{1}{2}\right)}{\Gamma(m)}=\sqrt{\frac{\pi \omega}{m}} \frac{\left(\begin{array}{c}m-\frac{1}{2} \\ m\end{array}\right) m !}{(m-1) !}=\sqrt{\pi m \omega}\left(\begin{array}{c}m-\frac{1}{2} \\ m\end{array}\right)$

and the variance as

$\operatorname{Var}[R]=\omega\left[1-\frac{1}{m} \frac{\Gamma^{2}\left(m+\frac{1}{2}\right)}{\Gamma^{2}(m)}\right]=\omega\left[1-\pi m\left(\begin{array}{c}\left.m-\frac{1}{2}\right)^{2} \\ m\end{array}\right]\right.$

Nevertheless, if we assume that such an algorithm will be in place from where we will be able to determine $m>0.5$ and $\omega$ from the computed mean and variance we can utilize the unified multipath probability density function. We only illustrate the logic below.

In order to account for another distribution our unified multipath distribution channel model, $f(r)$, can be modified as follows:

$$
f(r)=\sum_{i=1}^{4} n_{i} f_{i}(r),-\infty<r<\infty
$$

subject to

$$
\sum_{i=1}^{4} n_{i}=1,0 \leq n_{i} \leq 1
$$

Simply by changing the values of $n_{i}$ we can obtain an infinitely more number of distributions among the Nakagami with $m$ degrees of freedom, Lognormal, Rician, and Rayleigh than Nakagami with $m$ degrees of freedom alone; and therefore, there is a much higher chance that the actual, measurable multipath distribution which does not fit well the Nakagame with $m$ degrees of freedom distribution may fit well the unified geolocation channel model expanded among the Nakagami with $m$ degrees of freedom, Lognormal, Rician, and Rayleigh.

Future measured data will show the validity of this claim. The following section illustrates the computation of the observable paths.

\section{Observable Path Gain Computations ${ }^{\text {iii }}$}

Let assume that $a$ is the path gain which fits a Rayleigh distribution given by its probability density function (pdf)

$$
f_{A}(a)=\mid \begin{array}{cc}
\frac{a}{\lambda^{2}} e^{-\frac{1}{2}\left(\frac{a}{\lambda}\right)^{2}}, & a \geq 0 \\
0, & a<0
\end{array}
$$

and its cumulative probability distribution function (cdf)

$$
F_{A}(a)=\int_{-\infty}^{a} f_{A}(x) d x=1-e^{-\frac{1}{2}\left(\frac{a}{\lambda}\right)^{2}}
$$

where $\lambda$ is the parameter.

The LOS path gain is the expected value of the distribution which is given by

$$
a_{L O S}=E[a]=\int_{-\infty}^{\infty} a f_{A}(a) d a=\lambda \sqrt{\frac{\pi}{2}}
$$

The probability that the path gains; i.e., the NLOS gains will be smaller or equal to the LOS gain is equal to

$$
a_{N L O S \leq L O S}=\mid \begin{aligned}
& P\left[a \leq a_{L O S}\right] \\
& =F_{A}\left(a_{L O S}\right)
\end{aligned}=1-e^{-\frac{\pi}{4}}=0.5441
$$

In other words, the probability that the NLOS path gains is greater than the LOS path gain is

$$
a_{N L O S>L O S}=\mid \begin{gathered}
P\left[a>a_{L O S}\right] \\
=1-F_{A}\left(a_{L O S}\right)
\end{gathered}=e^{-\frac{\pi}{4}}=0.4559
$$

Let us assume that there exists a first reflection that has a gain of $3 \mathrm{~dB}$ greater than the LOS gain. The probability that the path gains will be smaller than the this gain is

$$
a_{N L O S \leq 3 d B L O S}=\mid \begin{gathered}
P\left[a \leq 3 d B a_{L O S}\right] \\
F_{A}\left(a_{L O S}\right)=1-e^{-\frac{1.99^{2} \pi}{4}}=0.9561
\end{gathered}
$$

On the other hand the probability that the NLOS path gain will be greater than the $3 \mathrm{~dB}$ path LOS gain is

$$
a_{N L O S>3 d B L O S}=\mid \begin{gathered}
P\left[a>3 d B a_{L O S}\right] \\
1-F_{A}\left(a_{L O S}\right)=e^{-\frac{1.99^{2} \pi}{4}}=0.0439
\end{gathered}
$$

If we were to convert this number in minutes we get 2.6 occurrences out of one minute. However, in order to get a better number for the observable path gain reflections we consider the following case. If we assume that the NLOS path gain will be greater than the $5 \mathrm{~dB}$ path gain above the LOS path gain than that probability is

$$
a_{N L O S>5 d B L O S}=\mid \begin{gathered}
P\left[a>5 d B a_{L O S}\right] \\
1-F_{A}\left(a_{L O S}\right)=e^{-\frac{10 \pi}{4}}=3.8820 e^{-4}
\end{gathered}
$$

This event occurs once in one hour. Again by the same token assuming a NLOS path gain greater than the $7 \mathrm{~dB}$ path gain above the LOS path gain than that probability is

$$
a_{N L O S>7 d B L O S}=\mid \begin{gathered}
P\left[a>7 d B a_{L O S}\right] \\
1-F_{A}\left(a_{L O S}\right)=e^{-\frac{10^{1.4} \pi}{4}}=2.7046 e^{-9}
\end{gathered}
$$

The occurrence of this event is one out of twelve years.

Similarly, it can be shown that the occurrence of an event for which the NLOS path gain will be greater than the $8 \mathrm{~dB}$ path gain above the LOS path gain is one out of $10^{7}$ years.

\section{Numerical, Theoretical Results}

Preliminary numerical, theoretical results section contains the first set of simulation results of: (1) indoor geolocation system 


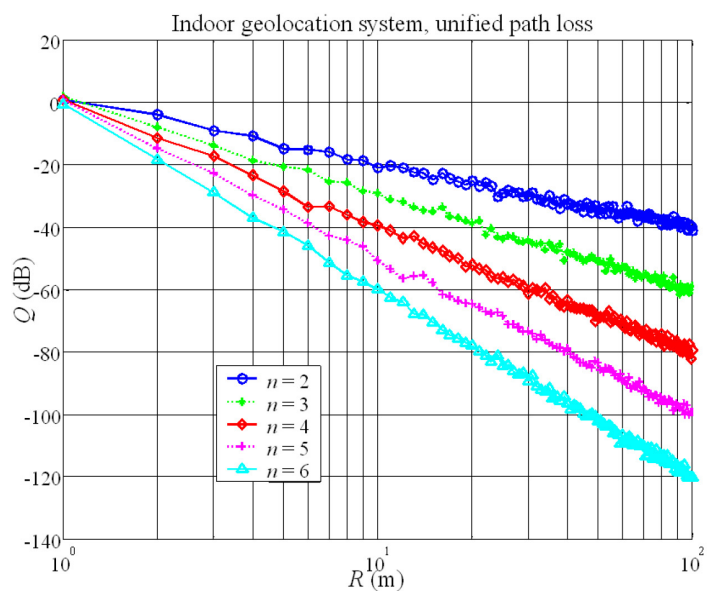

(a) Unified path loss (dB) vs. distance (m).

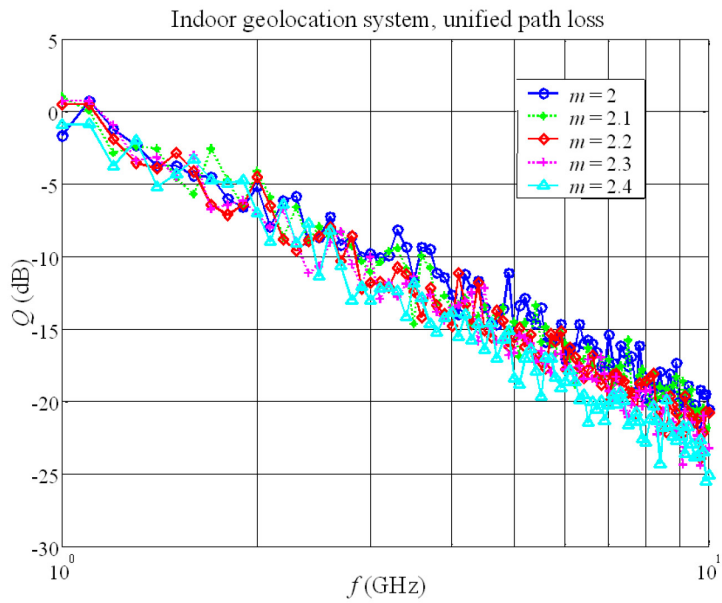

(b) Unified path loss (dB) vs. frequency $(\mathrm{GHz})$.

FIGURE 1: Unified path loss (dB) for a typical indoor geolocation system as a function of: (a) distance (m) and (b) frequency $(\mathrm{GHz})$ and $\sigma=1$.

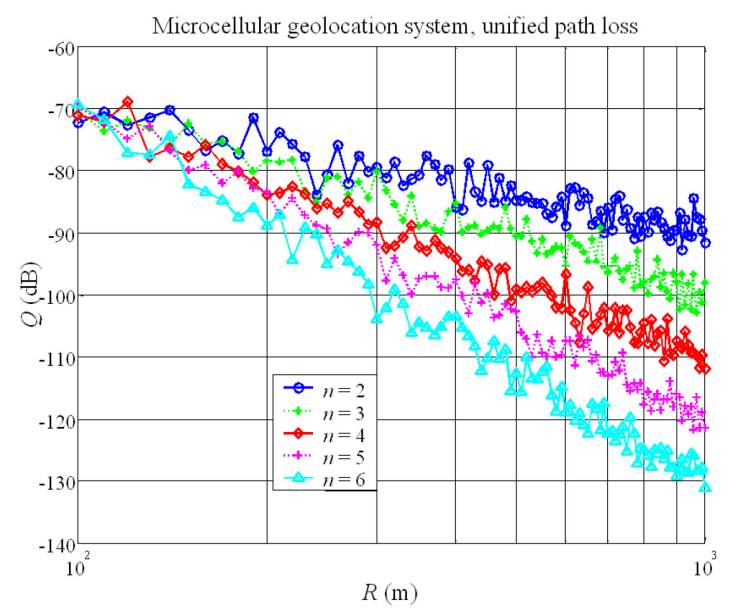

(a) Unified path loss (dB) vs. distance (m).

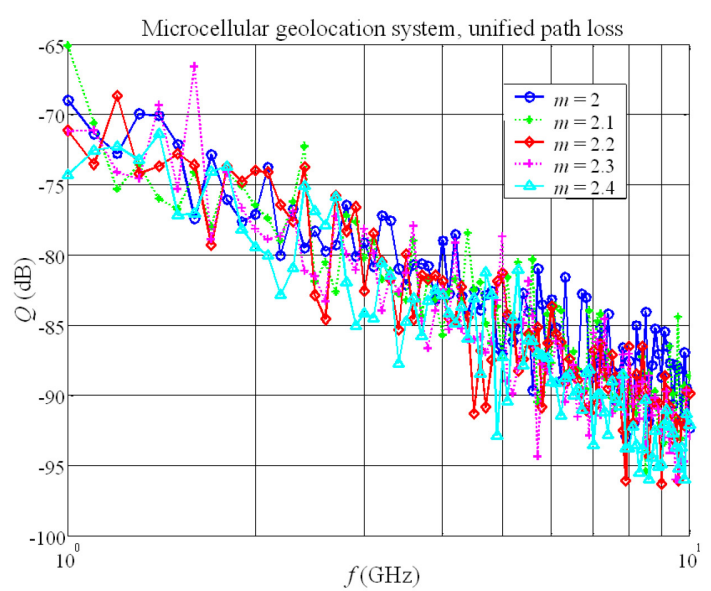

(b) Unified path loss $(\mathrm{dB})$ vs. frequency $(\mathrm{GHz})$.

FIGURE 2: Unified path loss $(\mathrm{dB})$ for a typical micro geolocation system as a function of: (a) distance (m) and (b) frequency $(\mathrm{GHz})$ and $\sigma=2$.

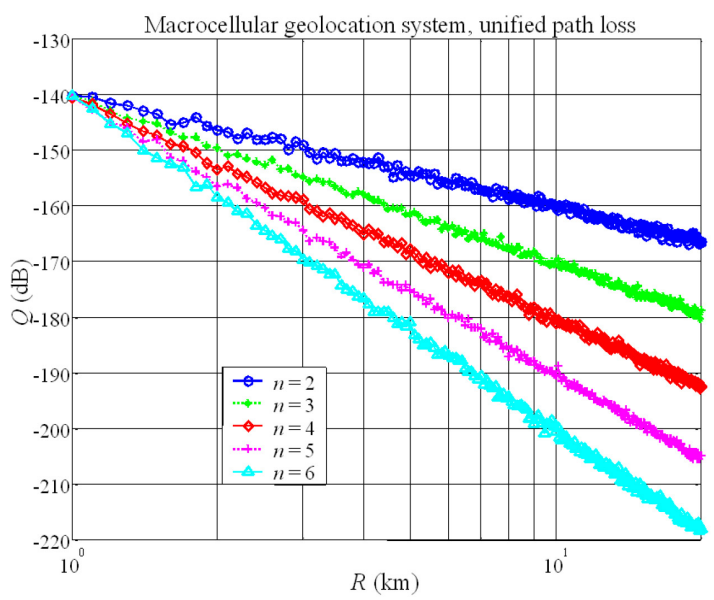

(a) Unified path loss (dB) vs. distance (m).

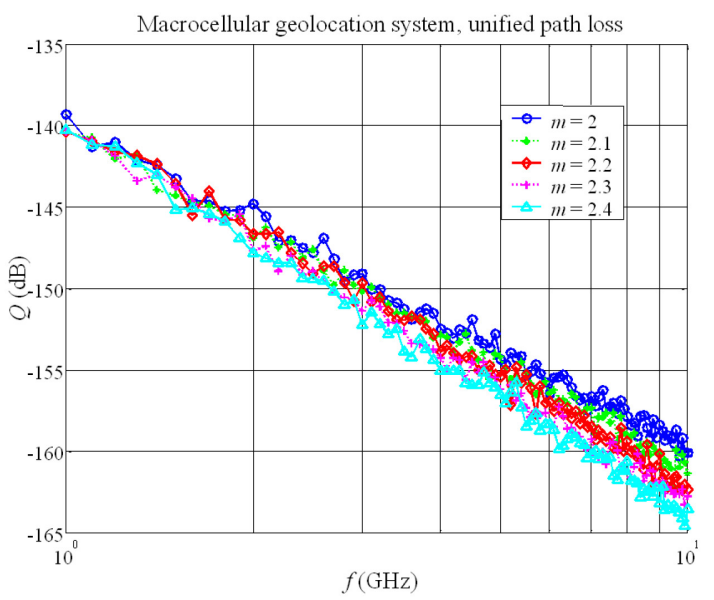

(b) Unified path loss $(\mathrm{dB})$ vs. frequency $(\mathrm{GHz})$.

FIGURE 3: Unified path loss $(\mathrm{dB})$ for a typical macro geolocation system as a function of: (a) distance (m) and (b) frequency $(\mathrm{GHz})$ and $\sigma=0.5$. 

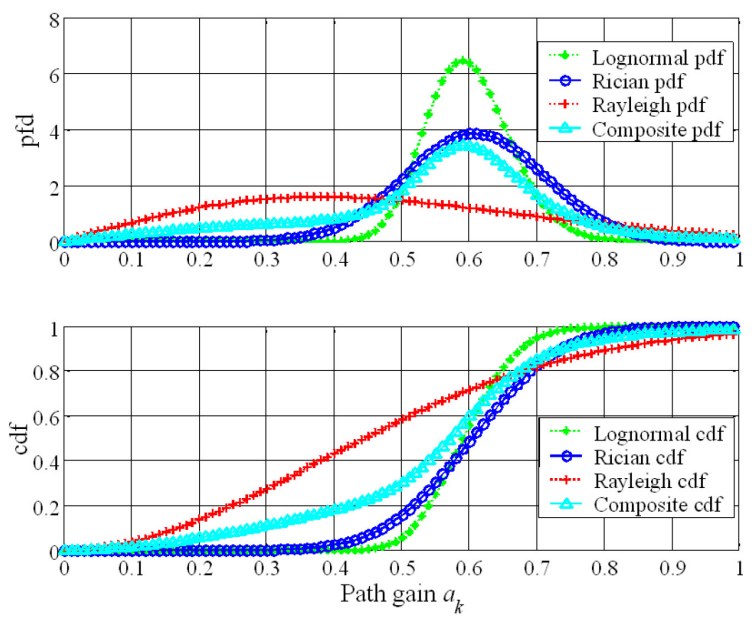

FIGURE 4: The composite cdf and pdf which contains a Lognormal, Rician, and Rayleigh fading channel for $\mu_{a}=0.6$ and $\sigma_{a}=0.0626$ and $n=\{0.25,0.35,0.4\}$.
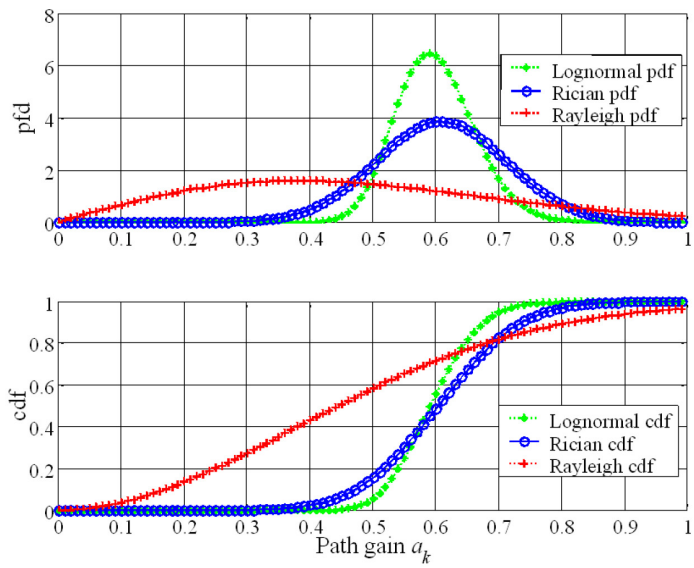

FIGURE 5: The cdf and pdf of a Lognormal, Rician, and Rayleigh fading channel for $\mu_{a}=0.6$ and $\sigma_{a}=0.0626$.

path loss; (2) micro geolocation system path loss, (3) macro geolocation system path loss, and (4) computation of the unified multipath distribution model.

\section{$5.1 \quad$ Indoor geolocation system path loss}

In this subsection we present, numerical results of the indoor geolocation system path loss using the exact closed form expression found in this paper and in Progri 2007 [1] and 2003 [2].

Figure 1 (a) illustrates the indoor geolocation system path loss for values of $R$ ranging from 1 to $100 \mathrm{~m}$, distance index of $n=\{2,3,4,5,6\}$ and $\sigma \sigma=1$. At $10 \mathrm{~m}$ distance between the transmitter and receiver the observed LOS path gain degradation ranges from $-20 \mathrm{~dB}$ to $-60 \mathrm{~dB}$. At $100 \mathrm{~m}$ however, these changes are in the range of $-40 \mathrm{~dB}$ to -120 $\mathrm{dB}$.

Figure 1 (b) illustrates the indoor geolocation system path loss for values of $f$ ranging from 1 to $10 \mathrm{GHz}$, frequency index of $m=\{2.1,2.2,2.3,2.4,2.5\}$ and $\sigma \sigma=1$. At $2 \mathrm{GHz}$ frequency the observed LOS path gain ranges from $-4.8 \mathrm{~dB}$ to $-7 \mathrm{~dB}$. At $10 \mathrm{GHz}$ however, these changes are in the range of $-20 \mathrm{~dB}$ to $-25 \mathrm{~dB}$.

Although the data presented here is not new, the interpretation of the data is new in the context of tying in our data with our ongoing research. The path loss caused by increase of the transmitter receiver distance is much more severe than the path loss caused by increase of the frequency of operation. The bottom line here is that we need to design future receivers or propose a signal structure that will account for 40 to $80 \mathrm{~dB}$ of signal degradation indoors.

\section{$5.2 \quad$ Micro geolocation system path loss}

In this subsection we present, numerical results of the micro geolocation system path loss using the exact closed form expression found in this paper and in Progri et al. 2007 [1] and 2003 [2].

Figure 2 (a) illustrates the micro geolocation system path loss for values of $R$ ranging from $100 \mathrm{~m}$ to $1 \mathrm{~km}$, distance index of $n=\{2,3,4,5,6\}$ and $\sigma \sigma=1$. At $300 \mathrm{~m}$ distance between the transmitter and receiver the observed LOS path gain degradation ranges from $-80 \mathrm{~dB}$ to $-100 \mathrm{~dB}$. At $1 \mathrm{~km}$ however, these changes are in the range of $-90 \mathrm{~dB}$ to $-130 \mathrm{~dB}$.

Figure 2 (b) illustrates the micro geolocation system path loss for values of $f$ ranging from 1 to $10 \mathrm{GHz}$, frequency index of $m=\{2.1,2.2,2.3,2.4,2.5\}$ and $\sigma \sigma=1$. At $2 \mathrm{GHz}$ frequency the observed LOS path gain ranges from $-74 \mathrm{~dB}$ to $-80 \mathrm{~dB}$. At $10 \mathrm{GHz}$ however, these changes are in the range of $-85 \mathrm{~dB}$ to $-95 \mathrm{~dB}$.

Although the data presented here is not new, the interpretation of the data is new in the context of tying in our data with our ongoing research. The path loss caused by increase of the transmitter receiver distance is much more severe than the path loss caused by increase of the frequency of operation.

\subsection{Macro geolocation system path loss}

In this subsection we present, numerical results of the macro geolocation system path loss using the exact closed form 
expression found in this paper and in Progri et al. 2007 [1] and 2003 [2].

Figure 3 (a) illustrates the macro geolocation system path loss for values of $R$ ranging from $1 \mathrm{~km}$ to $11 \mathrm{~km}$, distance index of $n=\{2,3,4,5,6\}$ and $\sigma=1$. At $3 \mathrm{~km}$ distance between the transmitter and receiver the observed LOS path gain degradation ranges from $-150 \mathrm{~dB}$ to $-170 \mathrm{~dB}$. At $11 \mathrm{~km}$ however, these changes are in the range of $-166 \mathrm{~dB}$ to -220 dB.

Figure 3 (b) illustrates the macro geolocation system path loss for values of $f$ ranging from 1 to $10 \mathrm{GHz}$, frequency index of $m=\{2.1,2.2,2.3,2.4,2.5\}$ and $\sigma=1$. At $2 \mathrm{GHz}$ frequency the observed LOS path gain ranges from $-145 \mathrm{~dB}$ to -148 dB. At $10 \mathrm{GHz}$ however, these changes are in the range of $-160 \mathrm{~dB}$ to $-165 \mathrm{~dB}$.

Although the data presented here is not new, the interpretation of the data is new in the context of tying in our data with our ongoing research. The path loss caused by increase of the transmitter receiver distance is much more severe than the path loss caused by increase of the frequency of operation.

\subsection{Computation of the unified multipath distribution model}

In this subsection we present, numerical results of the unified multipath distribution model using the exact closed form expression found in this paper and in Progri et al. 2007 [1] and 2003 [2].

Figure 4 illustrates the fact that the composite cdf and pdf which contains a Lognormal, Rician, and Rayleigh fading channel for $\mu_{a}=0.6$ and $\sigma_{a}=0.0626$ and $n=$ $\{0.25,0.35,0.4\}$.

Let's assume that the multipath distribution is either Lognormal or Rician.

Figure 5 shows the pdf and cdf of the Lognormal, Rician, and Rayleigh for the same mean $\mu_{a}=0.6$ and standard deviation $\sigma=0.0626$. If we assume that the first reflection path gain is $3 \mathrm{~dB}$ higher than the LOS path gain which is 0.6 then that gain will be 1.972 which is greater than 1 . For these gains both Lognormal and Rician distributions are under the Rayleigh distribution, which means that the tale probability that the NLOS path gain is greater than $3 \mathrm{~dB}$ than the LOS path gain is the smallest for the Lognormal distribution and the largest for the Rayleigh distribution; i.e., the following will hold
$a_{N L O S>7 d B L O S}=\mid \begin{gathered}P\left[a>7 d B a_{L O S}\right] \\ 1-F_{A}\left(a_{L O S}\right) \leq e^{-\frac{10^{1.4} \pi}{4}}=2.7046 e^{-9}\end{gathered}$

and this is the reason why the Rayleigh distribution is considered the most severe multipath distribution.

\section{Conclusions}

In conclusions we have revisit the unified geolocation channel model and have arrived at the following conclusions:

1. The unified path loss model to an extent represents the complete path loss model for all available geolocation systems. The bottom line here is that we need to design future receivers or propose a signal structure that will account for 40 to $80 \mathrm{~dB}$ of signal degradation indoors.

2. The unified multipath distribution not only presents the complete multipath distribution model but also provides the framework for all the computations required for observable path gains. However, one of the limitations is the ability to obtain specific distribution parameters such as in the case of Nakagami with $m$ degrees of freedom from the mean and value of the distribution resulting from the actual measurements. While Nakagami distribution may be a great distribution for power fading it is probably not a good distribution for gain fading for the reasons presented in the paper.

3. From the theoretical data it appears that reflections with gain either 3 , or 4 , or $5 \mathrm{~dB}$ or higher are on the order of either 2.6 , or 1.3 , or 1.4 occurrences out of either one minute, or three minutes, or one hour. On the other hand, reflections with gain either 6 or $7 \mathrm{~dB}$ or higher are of the order of 1 out of either three days or $\sim 12$ years. And this is the most important conclusion of this work that for simulation or implementation purposes we should never consider reflections with gains up to $7 \mathrm{~dB}$ greater than the LOS gain.

We are in lookout for experimental data to validate our models and also other distributions such as Weibull. Another approach could be that we modify our unified geolocation multipath distribution model to enable power calculations as opposed to gain calculations. This is also something that we will investigate and propose a new unified power multipath distribution model.

Since 2007 when this paper was originally presented a lot has occurred [13]. Although, this paper lacked a lot of mathematical background found in [13], it served as the bridge 
between the research that was initially published prior to 2007 [1]-[4], [15], [16] and the research that was published after 2007 [13].

\section{References}

[1] I., Progri, W.R., Michalson, J., Wang, M.C., Bromberg, "Theoretical data on support of a unified indoor geolocation channel model," in Proc. ION-NTM 2007, San Diego, CA, pp. 577-584, Jan. 2007.

[2] I.F. Progri, "An assessment of indoor geolocation systems," Ph.D. Dissertation, Worcester Polytechnic Institute, 408 pp, May 2003, URL: http://giftet.com/Progri/Progri_2003_05_Ph.D._Dissertati on.pdf.

[3] I.F. Progri, "A unified geolocation channel model--part I (path loss)," J. Geol. Geoinfo. Geointel., vol. 2017, article ID 2017071604, 15 pg., Nov. 2017. DOI: http://doi.org/10.18610/JG3.2017.071604.

[4] I. Progri, "A unified geolocation channel model--part II (multipath distribution)," in Proc. WTS-2006, Pomona, CA, pp. 1-8, Apr. 2006, DOI; http://doi.org/10.1109/WTS.2006.334542.

[5] H. Kuusniemi, "User level reliability and quality monitoring in satellite based personal navigation," Dr.Tech. Dissertation, Tampere University of Technology, Sep. 2005.

[6] A. Lakhzouri, E. Simona Lohan, I. Saastamoinen, and M. Renfors, "On second order statistics of the satellite-toindoor channel based on field measurements," in Proc. PIMRC 2005, Berlin, Germany, pp. 2632-2636, 9-14 Sep. 2005 ,

DOI: https://doi.org/10.1109/PIMRC.2005.1651920.

[7] A. Lakhzouri, E. Simona Lohan, I. Saastamoinen, and M. Renfors, "Interference and indoor channel propagation modeling based on GPS satellite signal measurements," in Proc. ION-GNSS 2005, Long Beach, CA, pp. 896-901, Sep. 2005.

[8] A. Ali, M. Kaveh, "Performance comparison of three different estimators for the Nakagami $m$ parameter using Monte Carlo simulation," in IEEE Commun. Letters, vol. 4, no. 4, pp. 119-121, April 2000, DOI: https://doi.org/10.1109/4234.841316.

[9] J. Luo, J. Zedler, “A statistical simulation model for correlated Nakagami fading channels," in Proc. WCC
2000 - ICCT 2000. (Cat. No.00EX420), Beijing, China, 2000, pp. 1680-1684 vol. 2, DOI: https://doi.org/10.1109/ICCT.2000.890981.

[10]J. Gubner, Probability and Random Processes for Electrical and Computer Engineers. NY: Cambridge University Press, 2006.

[11]Anon, "Gamma function," Wikipedia, the free encyclopedia, Nov. 2017, https://en.wikipedia.org/wiki/Gamma_function.

[12]Anon, "Nakagami distribution," Wikipedia, the free encyclopedia, Nov. 2017, https://en.wikipedia.org/wiki/ Nakagami_distribution.

[13]I. Progri, Indoor Geolocation Systems-Theory and Applications. Vol. I, $1^{\text {st }}$ ed., Worcester, MA: Giftet Inc., $\sim 800$ pp., 2017 (not yet available in print).

[14] I. Progri and W.R. Michalson, “An alternative approach to multipath and near-far problem for indoor geolocation systems," in Proc. 14 $4^{\text {th }}$ Inter. Tech. Mtg. Sat. Div. ION (ION GPS 2001), Salt Lake City, UT, pp. 1434-1443, Sep. 2001.

[15]I. Progri, W.R. Michalson, J. Wang, M.C. Bromberg, and R.J. Duckworth, "Requirements of a C-CDMA pseudolite indoor geolocation system," J. Geol. Geoinfo. Geointel., vol. 2017, article ID 2017071605, 5 pg., Nov. 2017. DOI: http://doi.org/10.18610/JG3.2017.071605.

[16] I. Progri, W.R. Michalson, J. Wang, M.C. Bromberg, "Indoor geolocation using FCDMA pseudolites: signal structure and performance analysis," Navigation, vol. 54, no. 3, pp. 242-256, fall 2007, DOI: http://doi.org/10.1002/j.2161-4296.2007.tb00407.x, URL: http://giftet.com/Progri/Progri_2007_fall_Navigation.pdf.

\section{Appendix A: Derivation of Nakagami Distribution Mean and Variance}

Because many book or articles do not derive the expected value (or the mean) and variance of the Nakagami distribution [1] [12] we provide a complete derivation of these two important statistical parameters.

From the definition of the expected value we have [10]

$$
E[R]=\int_{0}^{\infty} r f_{R}(r) d r=2\left(\frac{\mu}{\omega}\right)^{\mu} \frac{\int_{0}^{\infty} r r^{(2 \mu-1)} e^{-\frac{\mu}{\omega} r^{2}} d r}{\Gamma(\mu)}
$$

First, making the substitution $x=r^{2}$ we obtain

$$
E[R]=\left(\frac{\mu}{\omega}\right)^{\mu} \frac{\int_{0}^{\infty} x^{\mu-\frac{1}{2}} e^{-\frac{\mu}{\omega} x} d x}{\Gamma(\mu)}
$$


Second, making the substitution $y=\frac{\mu}{\omega} x$ yields

$E[R]=\left(\frac{\mu}{\omega}\right)^{\mu \int_{0}^{\infty}\left(\frac{\omega}{\mu}\right)^{\mu-\frac{1}{2}} \frac{\omega}{\mu} y^{\mu-\frac{1}{2}} e^{-y} d y}=\left(\frac{\omega}{\mu}\right)^{\frac{1}{2}} \frac{\int_{0}^{\infty} y^{\mu-\frac{1}{2}} e^{-y} d y}{\Gamma(\mu)}$

From where we obtain the final result which is also shown in (8) $[1],[12]$

$$
E[R]=\sqrt{\frac{\omega}{\mu}} \frac{\Gamma\left(\mu+\frac{1}{2}\right)}{\Gamma(\mu)} \mathrm{iv}
$$

Since we know the mean value, we can derive the variance, $\operatorname{Var}[R]$, from the definition [1], [12]

$$
\operatorname{Var}[R]=E\left[R^{2}\right]-(E[R])^{2}
$$

The second moment, $E\left[R^{2}\right]$, can be obtained using a similar expression from the first moment leaving the details to the reader we obtain:

$$
E\left[R^{2}\right]=\left(\frac{\omega}{\mu}\right) \frac{\Gamma(\mu+1)}{\Gamma(\mu)}=\left(\frac{\omega}{\mu}\right) \frac{\mu \Gamma(\mu)}{\Gamma(\mu)}=\left(\frac{\omega}{\mu}\right) \mu=\omega
$$

Hence, the variance, $\operatorname{Var}[R]$, can be obtained from [1] [12]

$$
\operatorname{Var}[R]=\omega-\frac{\omega}{\mu} \frac{\Gamma^{2}\left(\mu+\frac{1}{2}\right)}{\Gamma^{2}(\mu)}=\omega\left[1-\frac{\Gamma^{2}\left(\mu+\frac{1}{2}\right)}{\mu \Gamma^{2}(\mu)}\right]
$$

In general it can be shown that the $k$ th moment of the Nakagami distribution is equal to

$$
E\left[R^{k}\right]=\left(\frac{\omega}{\mu}\right)^{\frac{k}{2}} \frac{\Gamma\left(\mu+\frac{k}{2}\right)}{\Gamma(\mu)}
$$

This concludes the derivations of Nakagami distribution mean, variance, and its kth moment.

\section{Appendix B: Derivation of Inverse Nakagami Distribution}

In order to apply the unified multipath distribution theory we need to derive the inverse Nakagami Distribution. Since, this does not exist in any of the textbooks then it is even more useful to produce this information here.

Let assume that $R$ is a random variable, whose probability density function is Nakagami, is given by [8]-[10]:

\footnotetext{
i Signal structure is the arrangement of and relations between the parts or elements of the signal. Signal design is the plan or drawing produced to show the look and function or workings of the signal structure before it is produced and transmitted from the satellite or transmitter.

ii Ditto
}

$$
f_{R}(r)=\mid \begin{array}{cc}
2\left(\frac{\mu}{\omega}\right)^{\mu} \frac{1}{\Gamma(\mu)} r^{(2 \mu-1)} e^{-\frac{\mu}{\omega} r^{2}}, & r \geq 0 \\
0, & r<0
\end{array}
$$

Next, we define the transformation $W=g(R)=R^{-1}$ then the resulting transformation is

$$
f_{W}(w)=f_{R}\left(g^{-1}(w)\right)\left|\frac{d}{d w} g^{-1}(w)\right|
$$

The final expression of the Inverse Nakagami Distribution is

$$
f_{W}(w)=\mid \begin{array}{cc}
2\left(\frac{\mu}{\omega}\right)^{\mu} \frac{1}{\Gamma(\mu)} w^{-(2 \mu+1)} e^{-\frac{\mu}{\omega} w^{-2}}, & w \geq 0 \\
0, & w<0
\end{array}
$$

The cdf of the Inverse Nakagami Distribution is

$$
F_{W}(w)=\int_{-\infty}^{w} f_{W}(x) d x
$$

Leaving the details to the readers we can obtain the final expression of the cdf of the Inverse Nakagami Distribution as

$$
F_{W}(w)=\mid \begin{array}{cc}
\frac{\Gamma\left(\mu, \frac{\mu}{\omega} w^{-2}\right)}{\Gamma(\mu)}, & w \geq 0 \\
0, & w<0
\end{array}
$$

Next, he mean and the variance of the Inverse Nakagami Distribution are as follows:

$$
E[W]=\frac{\omega^{-\frac{1}{2}}}{\mu} \frac{\int_{0}^{\infty} u^{\mu-\frac{3}{2}} e^{-u} d u}{\Gamma(\mu)}=\left(\eta \equiv \sqrt{\frac{\mu}{\omega}}\right) \frac{\Gamma\left(\mu-\frac{1}{2}\right)}{\Gamma(\mu)}
$$

The second moment, $E\left[W^{2}\right]$, can be obtained using a similar expression from the first moment leaving the details to the reader we obtain:

$$
E\left[W^{2}\right]=\eta^{2} \frac{\Gamma(\mu-1)}{\Gamma(\mu)}=\eta^{2} \frac{1}{(\mu-1)}
$$

Hence, the variance, $\operatorname{Var}[W]$, can be obtained from

$\operatorname{Var}[W]=\eta^{2} \frac{1}{(\mu-1)}-\eta^{2} \frac{\Gamma^{2}\left(\mu-\frac{1}{2}\right)}{\Gamma^{2}(\mu)}=\eta^{2}\left[\frac{1}{\mu-1}-\frac{\Gamma^{2}\left(\mu-\frac{1}{2}\right)}{\Gamma^{2}(\mu)}\right]$

In general it can be shown that the $k$ th moment of the Inverse Nakagami distribution is equal to

$$
E\left[W^{k}\right]=\eta^{k} \frac{\Gamma\left(\mu-\frac{k}{2}\right)}{\Gamma(\mu)}
$$

This concludes the derivations of Inverse Nakagami distribution's pdf, cdf, mean, variance, and its kth moment.

\footnotetext{
iii There were a number of errors in this section which have been revised in this publication from the original publication in Progri et al. 2007 [1].

iv A recursive solution may be employed to produce the desired $\mu$ and $\omega$ from $E[R]$ and $\operatorname{Var}[R]$.
} 